\title{
ALMA Y CUERPO EN NUMENIO DE APAMEA
}

\author{
Gabriela Müller ${ }^{1}$ \\ Universidad de Buenos Aires (Argentina)
}

Recibido: 02.10.2018 - Aceptado: 24.11.2018

\begin{abstract}
RESUMEN
En el presente trabajo me propongo estudiar la relación entre el alma y el cuerpo en la filosofía de Numenio de Apamea, a partir del análisis de algunos fragmentos y testimonios conservados por diversas fuentes. Por una parte, mostraré cómo dichos materiales tienen como trasfondo común la polémica contra la filosofía de los estoicos. Por otra parte, intentaré argumentar que la concepción del alma como principio de estabilidad, por un lado, y como principio de movimiento, por otro, se resuelve en una diferenciación entre dos tipos de movimientos: uno que garantiza la estabilidad, el orden y la racionalidad de lo real y el otro que implica irracionalidad y desorden. Precisamente, en el encuentro e interacción entre ambos movimientos anímicos se ubican el mundo y también el hombre, en tanto ser corpóreo y animado.
\end{abstract}

Palabras clave: Numenio; alma; materia; cuerpo; ser.

\begin{abstract}
In this paper I will study the relationship between soul and body in Numenius of Apamea, by analysing some of the fragments and testimonies preserved by various sources. I will show how these texts have as a common background the controversy against the Stoics. On the other hand, I will argue that the conception of the soul as a principle of stability, on the one hand, and as a principle of movement, on the other, is resolved in a differentiation between two types of movements: one that guarantees the stability, the order and the rationality of the real and the other that involves irrationality and disorder. Precisely, in the encounter and interaction between both psychic movements are the world and the humans, as beings that are corporeal and animated.
\end{abstract}

Keywords: Numenius; soul; matter; body; being.

\footnotetext{
${ }^{1}$ gafermu@gmail.com
} 


\section{INTRODUCCIÓN}

El marcado dualismo antropológico y cosmológico, reflejado, en principio, en la distinción entre alma y cuerpo, es una nota central de la filosofía de Numenio de Apamea, tal como puede ser reconstruida a partir de los escasos fragmentos y testimonios que han llegado hasta nosotros a través de diferentes fuentes. Esto es en gran medida el resultado de una concepción dualista también en el nivel de los principios postulados por este filósofo: dios y materia. Sin embargo, este panorama esquemático resulta ser más complicado de lo que parece a primera vista: no sólo el principio divino aparece articulado en tres instancias (los llamados tres dioses, de entre los cuales el tercero podría ser identificado con el alma del mundo) sino que la materia es caracterizada como aquel principio de lo corpóreo y de lo irracional pero también es identificada con la causa del mal.

Por una parte, al abordar el estudio de los textos que permiten delimitar los conceptos de alma y cuerpo en la filosofía numeniana, nos topamos con un elemento común: la contraposición con las posiciones estoicas, que las propias fuentes que nos transmiten a Numenio (Eusebio de Cesarea, Nemesio de Émesa y Calcidio) se encargan de enfatizar. En este sentido, parece que la perspectiva estoica fue el horizonte polémico contra el cual este filósofo moldeó su propia concepción cosmológica y antropológica.

Por otra parte, el alma parece estar concebida de dos maneras diferentes: en tanto principio de estabilidad de lo corpóreo, el alma es el ser inmaterial que da estabilidad y consistencia a los cuerpos, que son entes materiales, y parece ser el principio opuesto a la materia, cuya naturaleza es el movimiento. Pero si entendemos al alma precisamente como principio de movimiento, se presenta entonces la necesidad de introducir un alma mala para explicar el movimiento desordenado con el que se identifica a la materia y que hace también que en el hombre haya un factor anímico irracional dependiente del principio material.

Dividiré mi trabajo siguiendo esta doble concepción del alma en tanto principio de estabilidad y en tanto principio de movimiento para ordenar los fragmentos analizados, pero procurando mostrar finalmente el modo en el que ambas caracterizaciones se relacionan entre sí para conformar una visión unitaria de la realidad que termina marcando una estrecha relación entre el alma y los principios de los cuales tanto lo corpóreo cuanto lo animado 
dependen. En este sentido, si para los estoicos dios y materia eran cuerpo, veremos en qué medida para Numenio ambos podrían ser caracterizados más bien como almas.

\section{EL ALMA COMO PRINCIPIO DE ESTABILIDAD DE LOS CUERPOS}

En el capítulo 17 del libro XV de la Praeparatio Evangelica (en adelante, PE), Eusebio de Cesarea cita dos fragmentos pertenecientes al tratado Sobre el bien


edición de Des Places. En ellos se establece una interesante relación entre los conceptos de "ser", "materia" y "cuerpo". Pero, como en una segunda versión del fragmento 4 trasmitida en el tratado De natura hominis de Nemesio de Émesa (el fragmento $4 \mathrm{~b}$ ), se introduce el concepto de alma, el análisis de estos textos me servirá como punto de partida para entender el modo en el que Numenio concibe la relación entre alma y cuerpo.

\section{a) Ser, materia y cuerpo: los fragmentos 3 y 4 a}

Los fragmentos 3 y 4a de Numenio están insertos en una sección del Libro XV de la $P E$ en la que Eusebio emprende una crítica a la filosofía estoica, ${ }^{2}$ cuya exposición desarrolla en los capítulos 14 y 15 mediante las citas del tratado Sobre la filosofía de Aristocles y del Epitome de Ario Dídimo. A partir del capítulo 16 y hasta el 22 Eusebio se ocupará de citar a filósofos platónicos que han criticado estas doctrinas estoicas. Así, a la cita de un breve pasaje del tratado de Porfirio Ad Boethum de anima en el capítulo 16 siguen, en el capítulo siguiente, los dos fragmentos de Numenio de los que nos ocuparemos. ${ }^{3}$

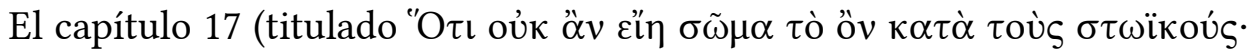

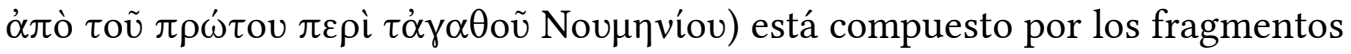


como lo indica el título del capítulo, se encargan de mostrar "que lo que es ( $\tau$ ò

2 Para una visión general de la estructura de este libro y las citas que allí aparecen, ver Des Places 1987, 15-25.

${ }^{3}$ Esta sección se completa con citas de Longino y de la Enéada IV 7 de Plotino, titulada

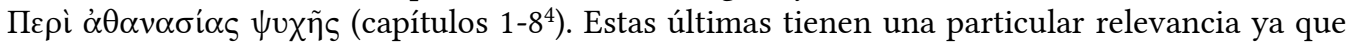
permiten completar una sección del texto que está ausente en la tradición manuscrita.

${ }^{4}$ Tanto en la edición de Des Places cuanto en las ediciones previas (la de Leemans y la de Thedinga), los fragmentos 3 y 4 a son insertados (y numerados correspondientemente) a continuación del fragmento 2 debido a que, por un lado, Eusebio nos dice explícitamente que tanto el fragmento 2 (citado en la sección "sobre el bien" del Libro XI) cuanto los fragmentos 3

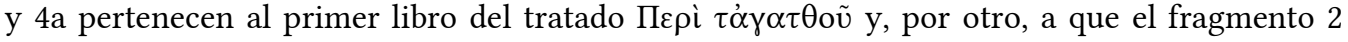

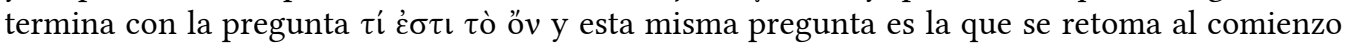
del fragmento 3 citado en este Libo XV. 
őv) no puede ser cuerpo $(\sigma \tilde{\omega} \mu \alpha)$ ", y esto está dicho por Numenio "contra los

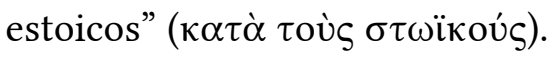

El pasaje comienza precisamente preguntando ¿qué es lo que es? ( $\tau$ í દ̇ $\sigma \tau \iota$ $\tau$ ò őv) y la respuesta a esta pregunta consiste, en primer lugar, en señalar la naturaleza incorpórea de lo que es ( $\tau$ ò őv): ${ }^{5}$

Pero, ¿qué es, entonces, lo que es?, ¿acaso estos cuatro elementos: la tierra, el fuego y las otras dos naturalezas intermedias?, ¿acaso ciertamente estas son las cosas que son, ya sea en general o al menos alguna de ellas individualmente? ${ }^{6}$

Numenio despliega su respuesta a la pregunta por lo que es distinguiendo dos posibilidades: que lo que es ( $\tau$ ò őv) se identifique con los cuatro elementos (tierra, fuego, agua y aire) tomados en su conjunto ( $\sigma \nu \lambda \lambda \dot{\eta} \beta \delta \eta \nu)$, o con alguno de ellos en particular ( $\left.\kappa \alpha \theta^{\prime \prime} \varepsilon v\right)$. Precisamente en el pasaje de Ario Dídimo citado

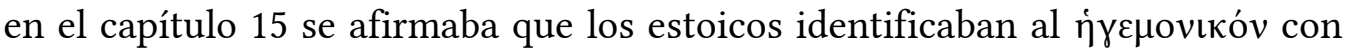
alguno de los elementos de la naturaleza. En cambio, ante esta pregunta, el interlocutor responde: ${ }^{7}$

- ¿Y cómo [podrían ser lo que es] las cosas que son no sólo generadas sino también recicladas $\left(\pi \alpha \lambda \iota v \alpha \rho^{\prime} \gamma \varepsilon \tau \alpha\right),{ }^{8}$ si por cierto es posible ver que estas cosas [los cuatro elementos] se generan unas a partir de otras y se transforman y no subsisten ni como elementos $(\sigma \tau o \iota \chi \varepsilon \tilde{\imath} \alpha)$ ni como compuestos $(\sigma \nu \lambda \lambda \alpha \beta \alpha i) ?^{9}$

Aquí el interlocutor avanza una primera caracterización de los elementos que impide que puedan ser identificados con lo que es: ellos se generan, se corrompen, cambian y no tienen una subsistencia (íđó $\tau \tau \alpha \sigma \iota \varsigma)$ ni en tanto elementos

\footnotetext{
${ }^{5}$ En un pasaje del Libro IX de la $P E$ (capítulo 7, 1 = fr. 1a) y en un testimonio de Orígenes (Contra Celsum I, 15 = fr. 1b) se indica que, según Numenio, la concepción de dios como incorpóreo es un punto en el cual coinciden diversas tradiciones religiosas: no sólo judíos y cristianos, sino también los brahmanes, los egipcios y los magos (probablemente, una alusión a los persas).

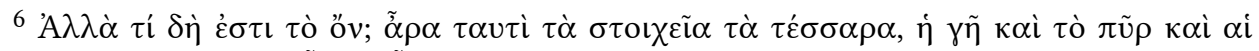

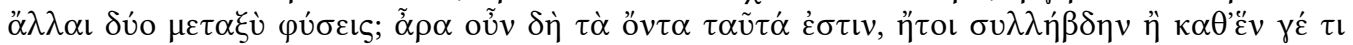
$\alpha \hat{\tau} \tau \tilde{\omega} v$; (fr. 3, 1-4). El texto griego y la numeración de los fragmentos de Numenio sigue la edición de Des Places 1973. Las traducciones en todos los casos son propias.

${ }^{7}$ Estos dos fragmentos se estructuran claramente en forma de preguntas y respuestas, lo

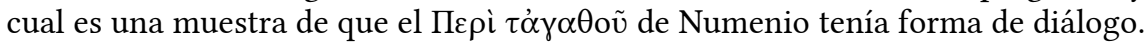

8 Sobre este término y su referencia al pasaje de Timeo 42e-43a, ver el comentario de Petty 2012, 120.

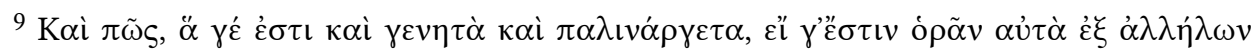

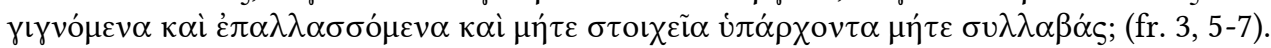


diferenciados ni en tanto compuestos a partir de esos elementos. Numenio

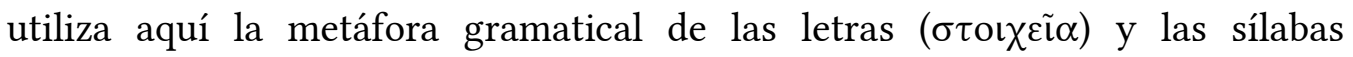
( $\sigma \nu \lambda \lambda \alpha \beta \alpha i ́)$, tomada sin duda de Timeo 48b8-c1, para mostrar de qué modo estos cuatro elementos pueden ser analizados individualmente o en relación con aquello que componen. Pero, en ambos casos, se encuentran sometidos a la generación, la corrupción, el cambio y la transformación mutua.

A esta caracterización sigue una identificación de estos elementos con los cuerpos $(\sigma \omega \dot{\mu} \alpha \tau \alpha)$. Dice Numenio:

- Ciertamente, en tanto cuerpo, estas cosas [los cuatro elementos] no serían, de este modo, lo que es. Pero, entonces, ¿estas cosas no [son lo que es], pero la materia sí puede ser lo que es? ${ }^{10}$

Numenio niega que los elementos puedan ser lo que es ( $\tau$ ò őv), puesto que son cuerpo $(\sigma \tilde{\omega} \mu \alpha)$. Pero luego se pregunta si la materia (Ű $\lambda \eta)$ puede ser lo que es. Parece, entonces, que Numenio traza una distinción entre $\sigma \tilde{\omega} \mu \alpha$ y $\ddot{\nu} \lambda$, que va en contra de la identificación de estas dos nociones en la filosofía estoica, tal como se señalaba en el pasaje de Aristocles citado por Eusebio en el capítulo 14 del Libro XV de la $P E$, donde se afirmaba que dios y materia eran cuerpo. En cambio, para Numenio, si en el caso de los cuerpos la identificación con lo que es no era posible, hay que investigar ahora si es posible en el caso de la ü $\lambda \eta$. La respuesta será también negativa, puesto que el fragmento termina con una caracterización de la materia que le impedirá ser identificada con lo que es. Dice Numenio:

- Pero también es imposible con toda seguridad que ella [sea lo que es] a causa de su incapacidad de permanecer; pues la materia es como un río de corriente rápida e inestable, indefinida e interminable en profundidad, anchura y longitud. ${ }^{11}$

La permanencia es para Numenio una de las características más propias de lo

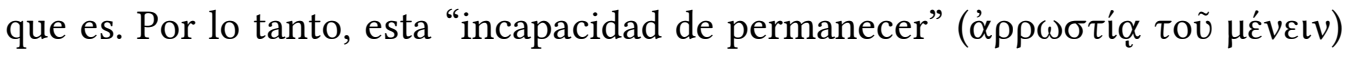
de la materia la excluye del ámbito de lo que es ( $\tau$ ò őv). Por otra parte, la imagen de la corriente rápida del río es utilizada por Numenio para mostrar la indeter-

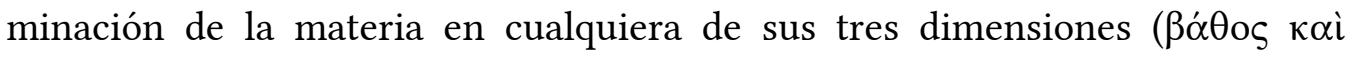

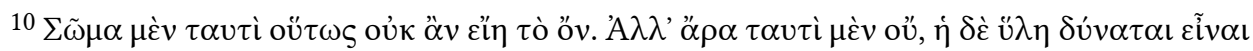
őv; (fr. 3, 8-9). Cabe notar la ausencia del artículo en este último őv; de todos modos, traduzco por "lo que es".

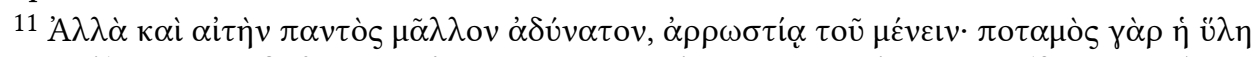

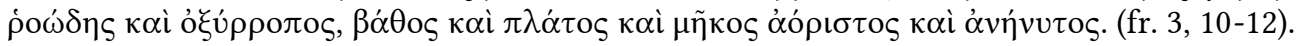




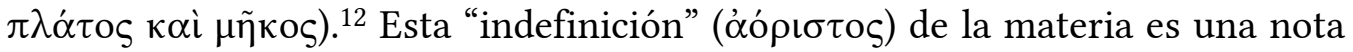
característica que veremos reaparecer en el fragmento siguiente. ${ }^{13}$

La caracterización de la materia que Numenio realiza en el fragmento 3 es desplegada en el fragmento 4a mediante una concatenación conceptual, que va relacionando una serie de notas que le son propias. Dice Numenio:

De modo que el argumento (入óyos) ha hablado correctamente diciendo que, si la materia es ilimitada, ella es indefinida; si es indefinida, [es] irracional; si es irracional, [es] incognoscible. Pero siendo incognoscible es necesario que ella sea carente de orden, puesto que lo que está ordenado sería por cierto muy fácil de conocer. Pero lo carente de orden no está firme y lo que no está firme no puede ser lo que es. Pero esto era lo que habíamos convenido entre nosotros mismos en lo anterior: que es impío que todas estas cosas le convengan a lo que es. ${ }^{14}$

El $\lambda$ óyos que Numenio desarrolla aquí va enlazando una tras otra una serie de

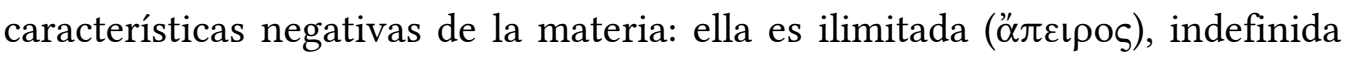
(’ó ( $\alpha \tau \alpha \kappa \tau \circ \varsigma)$. Para probar la relación entre estas dos últimas notas parece necesario un breve argumento suplementario: lo que es incognoscible carece de orden, dado que lo ordenado, lo que tiene $\tau \dot{\alpha} \xi ı \varsigma$, es fácil de conocer. Demostrar este último punto, es decir, que la materia carece de orden, es importante puesto que permite llegar a la última nota: la materia no está firme, no permanece (oủ $\chi$ $\check{\varepsilon} \sigma \tau \eta \kappa \varepsilon v$ ). Puesto que esta es la característica que le conviene a lo que es ( $\tau$ ò őv) principalmente, ${ }^{15}$ y la materia carece de esta permanencia requerida, ella no puede ser lo que es ( $\tau$ ò őv). Y esto era justamente lo que se había convenido antes: tal vez se trate de una referencia al pasaje final del fragmento 3 donde, de hecho, ya se había dicho que la materia no podía ser lo que es debido a su incapacidad para permanecer. Si en ese fragmento esto se explicaba en términos

12 La misma imagen aparece también en el fragmento 18 de Numenio.

13 Des Places 1973, 215-223 analiza estos fragmentos en relación con el fragmento 11 en

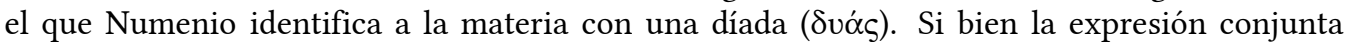

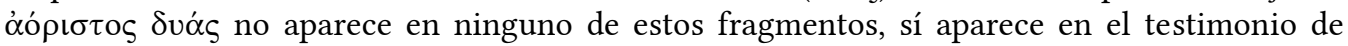
Calcidio (fr. 52).

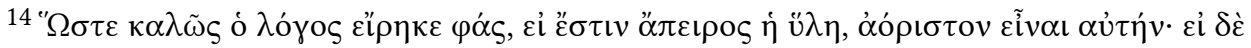

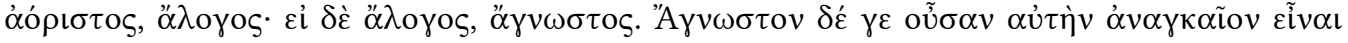

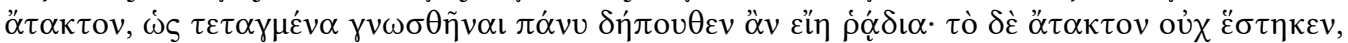

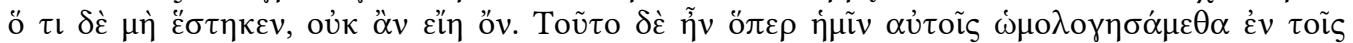

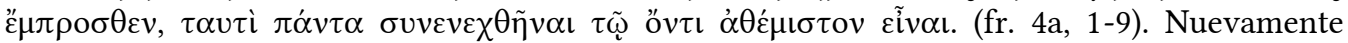
aparece el öv sin artículo en la línea 7; nuevamente, traduzco por "lo que es".

${ }^{15}$ En los fragmentos 5 y 8, citados también por Eusebio pero en el Libro XI de su $P E$, se

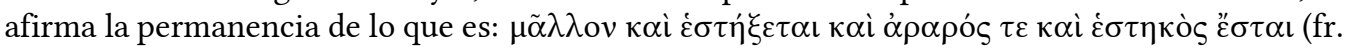

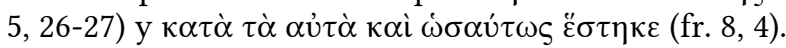


algo poéticos, mediante la imagen del río y su corriente, aquí aparece un $\lambda$ ó $\gamma$ o que lleva a la misma conclusión. Pero este $\lambda$ óyoৎ está expresado en términos condicionales y parte de una suposición que, precisamente, está justificada por la imagen del río: la materia es interminable ( $\alpha v \eta ́ v v \tau o \varsigma)$ en profundidad, anchura y longitud, es decir, es ilimitada (״̈ $\pi \varepsilon \iota \rho \circ \varsigma)$.

El interlocutor, a continuación, acuerda con este $\lambda$ ójos. Dice:

- Que parezca preferentemente a todos; pero, si no [a todos], por lo menos a mí.

- Ciertamente digo que la materia, ni ella misma ni los cuerpos, es lo que es. ${ }^{16}$

Numenio insiste en negar que tanto la materia cuando los cuerpos puedan ser lo que es ( $\tau$ ò őv), lo cual no hace más que reforzar la distinción entre $\sigma \tilde{\omega} \mu \alpha$ y ű $\lambda \eta$ que ya aparecía en el fragmento anterior. Luego de llegar a este acuerdo, se plantea una nueva posibilidad para responder a la pregunta qué es lo que es. Dice el interlocutor:

- ¿Y qué, entonces?, ¿acaso tenemos en la naturaleza del universo alguna otra cosa más allá de estas?

- Sí. Decir esto no es nada complicado, si primero, mientras dialogamos entre nosotros mismos, a la vez, lo intentamos. Puesto que los cuerpos por naturaleza están muertos y son cadáveres y se desplazan y no permanecen en lo mismo, ¿acaso no era necesario [algo] que los sostuviera?

- Con toda seguridad.

- Si no sucediera esto, ¿acaso permanecerían?

- En absoluto. ${ }^{17}$

Que debe existir otra cosa, además de los cuerpos y la materia, se demuestra mediante la caracterización de los cuerpos ( $\tau \dot{\alpha} \sigma \omega ́ \mu \alpha \tau \alpha)$ como cadáveres sin vida

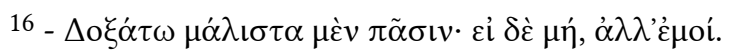

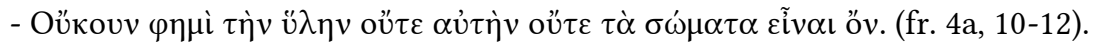

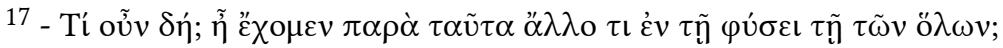

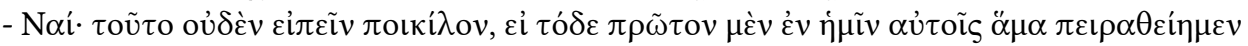

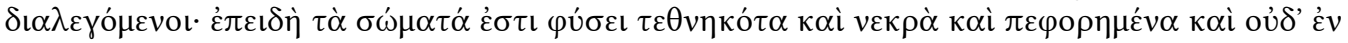



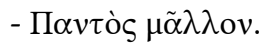

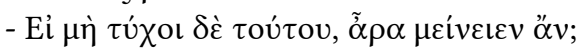

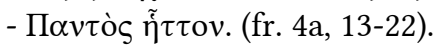




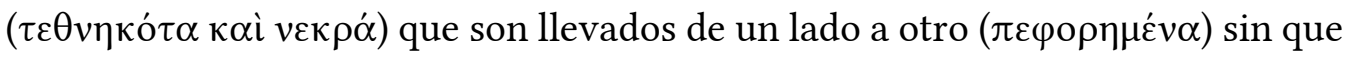

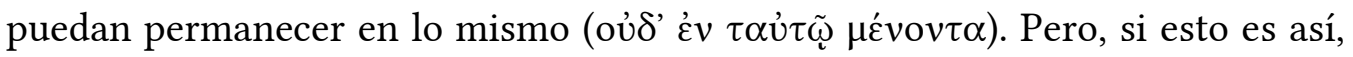
entonces es necesario que tengan algo que los sostenga ( $\kappa \alpha \theta \dot{\varepsilon} \xi o v)$, que los ancle, para lograr algún tipo de permanencia "en lo mismo" ( $\dot{\varepsilon} v \tau \alpha \dot{\tau} \tau \tilde{\omega})$. Cabe notar en este punto que Numenio utiliza el verbo $\kappa \alpha \tau \varepsilon ́ \chi \omega$ para referirse a este "sostenimiento" que es necesario en el caso de los cuerpos. En cambio, el texto de Ario Dídimo citado en el capítulo 15 de este Libro XV, mediante el cual se exponía la

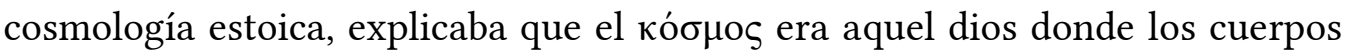
( $\tau \grave{\alpha} \sigma \omega ́ \mu \alpha \tau \alpha)$ estaban "contenidos"; y allí se utilizaba el verbo $\pi \varepsilon \rho \iota \varepsilon ́ \chi \omega$, que, por

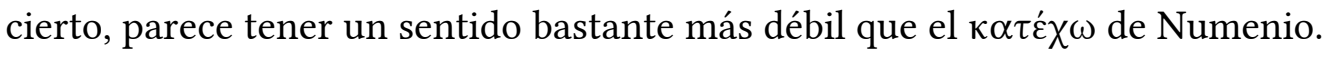

A diferencia de la materia cuya "incapacidad para permanecer"

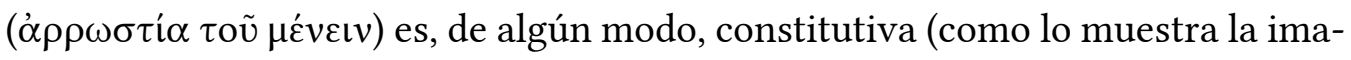
gen del río del fragmento 3), según Numenio, en el caso de los cuerpos sí es necesario algo que los cohesione, que los sostenga "en lo mismo" ( $\dot{\varepsilon} \nu \tau \alpha \dot{v} \tau \tilde{\omega}) \mathrm{y}$ los haga ser eso que son en cada caso. Si no existiera esto, parecería que no podríamos hablar propiamente de $\sigma \tilde{\omega} \mu \alpha$, ya que estaríamos precisamente ante la materia totalmente indefinida.

Numenio se ocupará, en lo que sigue de este fragmento, de indicar por

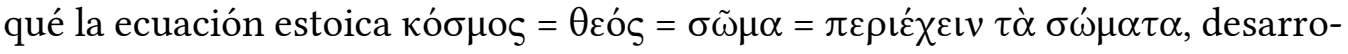
llada en el capítulo 15 a través del testimonio de Ario Dídimo, no es aceptable. Y para ello mostrará la necesidad de postular algo incorpóreo que oficie de principio de “contención” o "sostén” de los cuerpos. Dice Numenio:

Por lo tanto, ¿qué es lo que los sostiene ( $\tau$ ò $\kappa \alpha \tau \alpha \sigma \chi \eta \tilde{\sigma} \sigma o v)$ ? Si, por un lado esto también fuera cuerpo, me parece que esto, puesto que se disuelve y se dispersa, estaría necesitado de un Zeus Salvador. Por el contrario, si es necesario que esto se libere de la afección de los cuerpos para que pueda apartar la destrucción de aquellos que

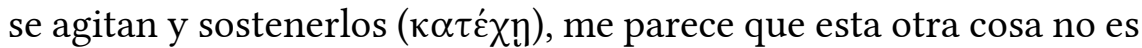
sino sólo lo incorpóreo. Pues ciertamente de entre todas las naturalezas, ella sola está firme y es estable y para nada corpórea. Ciertamente ni se genera, ni crece, ni se mueve respecto de ningún otro movimiento, y por eso parece bien justo [afirmar] que lo incorpóreo es superior. ${ }^{18}$

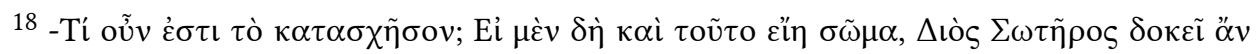

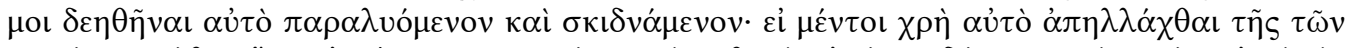

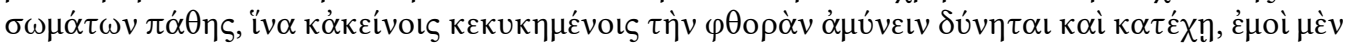

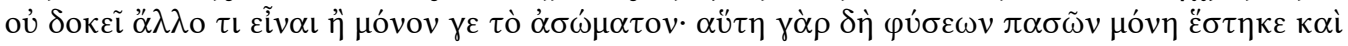

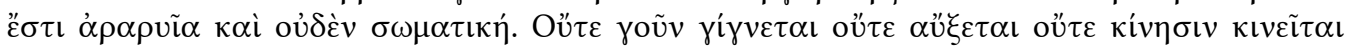

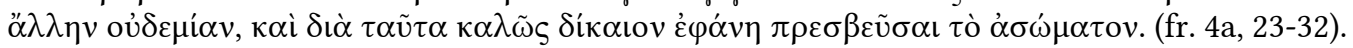


El razonamiento que se despliega en este pasaje es el siguiente: si lo que sostiene a los cuerpos fuera también un cuerpo, como plantean los estoicos, entonces también esto estaría sujeto a la misma dispersión de los cuerpos y, por lo tanto, estaría necesitado, a su vez, de algo que lo sostuviera y así se iría al infinito. La única solución en este caso, dice Numenio irónicamente, sería postular un Zqùs $\Sigma \omega \tau \eta ́ \rho$ que, cual deus ex machina, cortara esta regresión infinita y evitara la absoluta dispersión que implicaría la destrucción o corrupción ( $\varphi \theta 0 \rho \alpha ́)$ de los cuerpos. Pero la manera adecuada de que no se produzca esta regresión es admitiendo que esto que "sostiene" a todo lo corpóreo no debe padecer la misma afección $(\pi \alpha \dot{\alpha} \theta \eta)$ de los cuerpos, es decir, no debe ser un cuerpo, sino algo otro

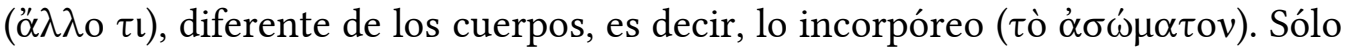

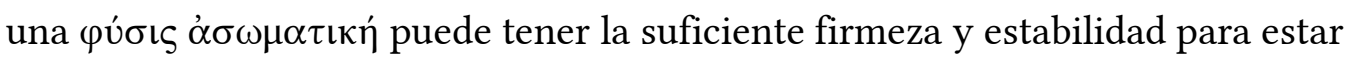
desligada de todo movimiento y cambio y poder oficiar de "sostén" de los cuerpos.

Reaparecen también en este pasaje las nociones de firmeza y estabilidad

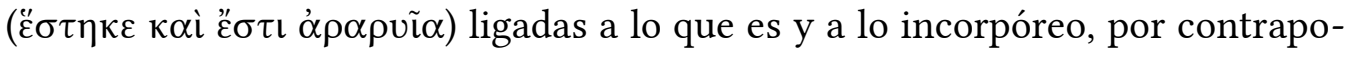
sición a la generación, el crecimiento y el movimiento al que están sometidas las cosas corpóreas. Y el fragmento concluye señalando la prioridad de lo incorpóreo respecto de los cuerpos. Lo incorpóreo es anterior, superior $(\pi \rho \varepsilon \sigma \beta \varepsilon \tilde{v} \sigma \alpha \mathrm{l})$, ya que los cuerpos necesitan de lo incorpóreo como este "sostén" o "anclaje" que los conserva y los hace ser eso que son. Y, por este motivo, no llama la atención que esto incorpóreo se haya identificado con $\tau$ ò őv.

El análisis de estos dos fragmentos nos deja, por un lado, ante una contraposición entre materia y cuerpo, por un lado, y lo que es y lo incorpóreo, por otro. Pero también nos muestra una distinción entre la materia y los cuerpos: la primera es totalmente indeterminada y es caracterizada sólo en términos negativos, los segundos, en cambio, están "sostenidos" por lo incorpóreo. Respecto de esta distinción entre materia y cuerpo es interesante notar cómo el fragmento parece estar retomando la noción aristotélica de materia próxima y los niveles de determinación de la materia distinguidos en Metafísica H5, 1044b341045a6: en efecto, la caracterización de los cuerpos como cadáveres ( $v \varepsilon \kappa \rho \alpha ́)$ sirve a Numenio como punto de partida de un argumento que concluye en la necesidad de un sostén incorpóreo que impida la corrupción ( $\varphi \theta 0 \rho \alpha ́)$ corpórea. En definitiva, todo cuerpo, para no ser algo muerto ni mera materia indeterminada, debe poseer ya un grado de determinación formal de modo tal que pue$\mathrm{da}$, como veremos a continuación en el fragmento $4 \mathrm{~b}$, ser caracterizado como

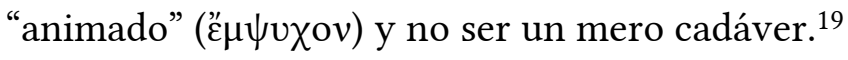

19 Agradezco a Gabriela Rossi por hacerme notar esta posible conexión con Aristóteles, que seguramente valdrá la pena explorar más en detalle en algún trabajo futuro. Asimismo, me permito remitir al artículo de Fabián Mié "Refining the Material Substance: Aristotle's Program in Metaphysics H1-5", publicado en este mismo volumen y que contiene una esclarecedora explicación de este pasaje aristotélico. 


\section{b) Alma, cuerpo y materia: el fragmento $4 \mathrm{~b}$}

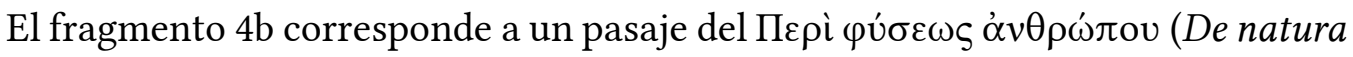
hominis), un tratado de antropología filosófica compuesto por Nemesio de Émesa. ${ }^{20}$ El segundo de los 44 capítulos que lo conforman está dedicado al alma


Nemesio comienza estableciendo una primera distinción entre aquellos que concibieron al alma como un cuerpo $(\sigma \tilde{\omega} \mu \alpha)$ y aquellos que consideraron que es incorpórea ( $\alpha \sigma \omega \dot{\alpha} \mu \tau \sigma \varsigma)$ ). Demócrito, Epicuro y los estoicos son ubicados en el primer grupo, aunque sus posiciones difieren respecto de la ov̉ $\sigma i ́ \alpha$ de esta alma corpórea. Dentro del segundo grupo, Nemesio menciona las definiciones del alma que han dado Tales, Pitágoras, Platón, Aristóteles y Dinarco. Luego se refiere a los Maniqueos, para quienes existe un alma única para todas las cosas, y los diferencia de un segundo grupo, quienes sostienen que hay muchas almas que difieren según su especie, y de un tercer grupo que combina ambas doctrinas diciendo que hay un alma única y hay muchas almas particulares.

Inmediatamente, luego de este rápido recorrido por las distintas posiciones sobre el alma según su cualidad ( $\sigma \tilde{\omega} \mu \alpha$ - $\alpha \sigma \omega ́ \mu \alpha \tau$ os) y según su cantidad

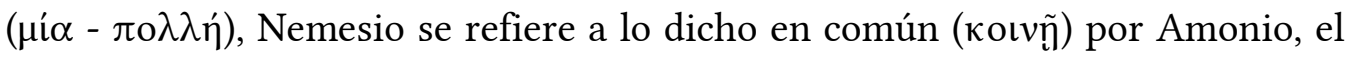
maestro de Plotino, y por Numenio, el pitagórico, contra todos aquellos que sostuvieron que el alma es cuerpo $(\sigma \tilde{\omega} \mu \alpha) .{ }^{21}$ Debemos tener en cuenta, por lo tanto, que estamos ante un testimonio, que se refiere a lo dicho no sólo por Numenio sino también por Amonio (a diferencia del fragmento 4a en el que Eusebio cita las palabras de Numenio). De hecho, es probable que el argumento desarrollado aquí se tratara de un argumento general compartido por las filosofías anti-materialistas, entre las que el pitagorismo y el platonismo eran las más destacadas. ${ }^{22}$ Nemesio pudo haberse basado en el texto de Numenio que Eusebio ha citado (el fragmento 4a), en cuyo caso las diferencias entre ambos textos pueden deberse a interpretaciones propias. Pero también es posible que

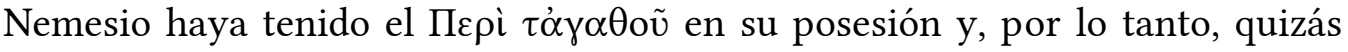
estos elementos novedosos estaban en efecto presentes en la obra de Numenio. ${ }^{23}$

El pasaje comienza diciendo:

Los cuerpos que por su naturaleza propia son cambiantes y dispersos y siempre divisibles al infinito, no quedando en ellos nada inmutable, necesitan de [algo] que los cohesione ( $\left.\sigma v v^{\prime} \chi 0 \nu\right)$ y los

20 Para una presentación general de este autor y su obra, ver Barnard 1972, 254-270.

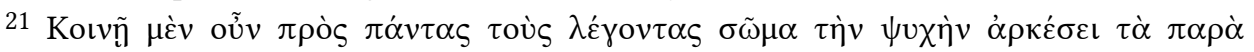


(Fr. 4b, 1-4).

22 Dodds 1960, 25.

${ }^{23}$ Para un análisis detallado de este textos, ver Dörrie-Baltes 2002, 180-184. 


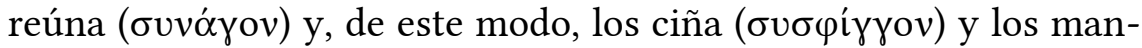
tenga unidos ( $\sigma$ $\gamma \kappa \rho \alpha \tau$ ○ṽv), a lo que precisamente llamamos alma. Por lo tanto, si el alma es un cuerpo de tal o cual clase, aunque sea compuesto de las partículas más pequeñas, ¿qué es lo que, a su vez,

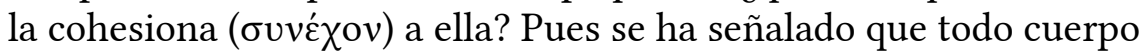
necesita de [algo] que lo cohesione ( $\sigma v v \varepsilon ́ \chi o v)$, y así [se llega] al infinito, hasta que arribemos a [algo] incorpóreo $(\dot{\alpha} \sigma \dot{\omega} \mu \alpha \tau o v){ }^{24}$

En esta primera parte, se despliega el mismo argumento que en el fragmento 4a, quizás expuesto de un modo más claro y ordenado. Sin embargo, podemos observar algunos cambios. En primer lugar, la caracterización de los cuerpos ( $\tau \grave{\alpha} \sigma \omega ́ \mu \alpha \tau \alpha)$ agrega, a la inestabilidad y dispersión, la idea de la divisibilidad al

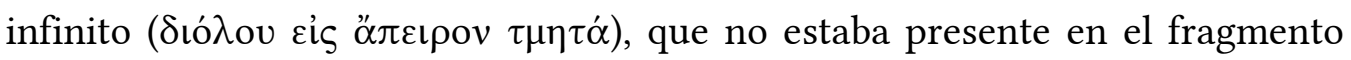
citado por Eusebio. En segundo lugar, el verbo $\kappa \alpha \tau \dot{\varepsilon} \chi \varepsilon \iota \nu$ es reemplazado por

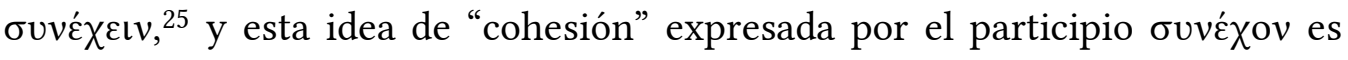
reforzada por otros tres participios que poseen el prefijo $\sigma v v-$ : $\sigma u v \alpha ́ \gamma o v$,

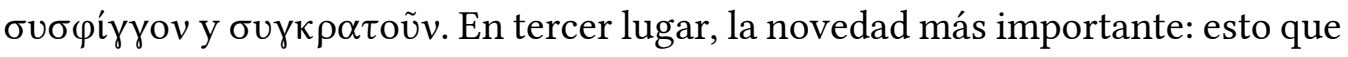
"cohesiona" y "ciñe" a los cuerpos es el alma ( $\psi v \chi \eta ́)$. Finalmente, este pasaje menciona la doctrina estoica de que el alma es un cuerpo constituido de partículas sutiles. Incluso en ese caso (y, quizás, más aún en ese caso), necesita de un principio que la contenga y que le dé unidad. Nemesio aquí explicita una idea que estaba implícita en el fragmento 4a: esta doctrina estoica produce un

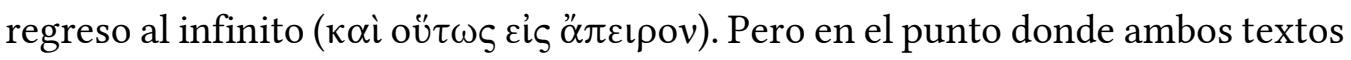
(frags. 4a y 4b) coinciden es en la necesidad de que esto que "sostiene" ( $\kappa \alpha \tau \dot{\varepsilon} \chi \omega)$ o "cohesiona" ( $\sigma u v \varepsilon ́ \chi \omega)$ a los cuerpos, para evitar el regreso al infinito o tener que apelar a un Zzùs $\Sigma \omega \tau \eta \dot{\rho}$, sea incorpóreo ( $\alpha \sigma \omega \dot{\mu} \mu \alpha \tau o v)$.

El pasaje continúa con una doctrina, adjudicada explícitamente a los estoicos, que intenta eludir esta crítica. Dice Nemesio:

Pero si dijeran, como los estoicos, que hay en torno a los cuerpos

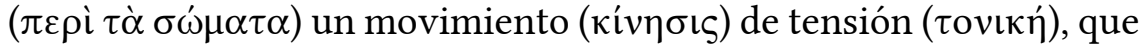
se mueve hacia adentro y al mismo tiempo hacia afuera, y que uno,

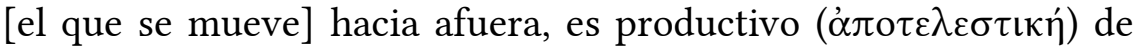
magnitudes y cualidades, y el otro, [el que se mueve] hacia adentro,

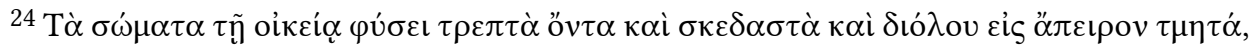

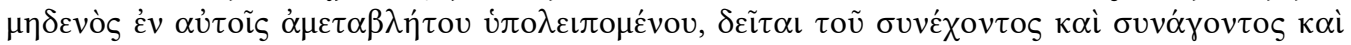

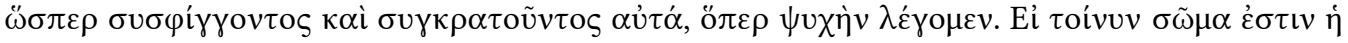

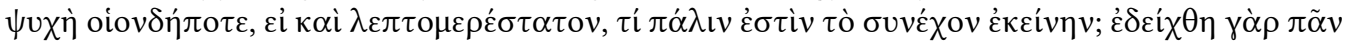

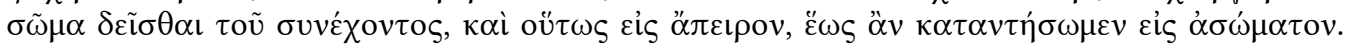
(Fr. 4b, 5-13).

${ }^{25}$ Este nuevo vocabulario tiene claras resonancias estoicas: en efecto, la noción de "causa

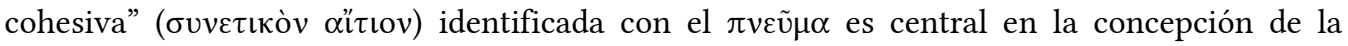
causalidad del estoicismo. Ver SVF II 344-351, 356 y 439-440 y el comentario de Boeri-Salles 2014, 348-360. 


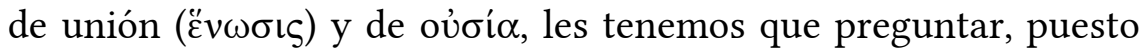

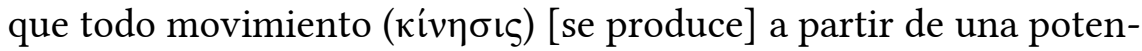

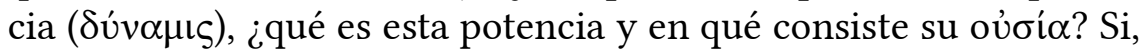
por un lado, ciertamente [responden que] esta potencia [es] alguna materia ( $\ddot{\prime} \lambda \eta \tau \iota \varsigma)$, utilizaré de nuevo los mismos argumentos ( $\lambda$ óyoı). Si, por otro, [responden que] no [es] materia (ü $\lambda \eta)$ sino material ( $\varepsilon v \cup \lambda \circ v$ ) (y lo material es diferente respecto de la materia; pues se dice que lo material es lo que participa ( $\mu \varepsilon \tau \dot{\varepsilon} \chi 0 v)$ de la materia), entonces luego [preguntaré] ¿qué es lo que participa de la materia?, ¿esto es materia (ü $\lambda \eta$ ) o es inmaterial ("ळü $\lambda$ ov)? Si, por un lado, [es] materia, ¿cómo [es] material y no materia? Si, por otro, no [es] materia, entonces [es] inmaterial; pero, si es inmaterial, no es cuerpo, pues todo cuerpo es material. ${ }^{26}$

En esta parte del texto se apela a la doctrina estoica de que los cuerpos tienen

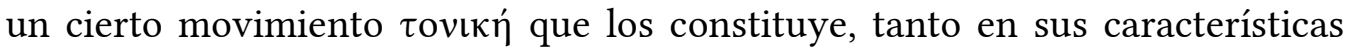


refutación de esta tesis consiste en hacer depender este movimiento de una

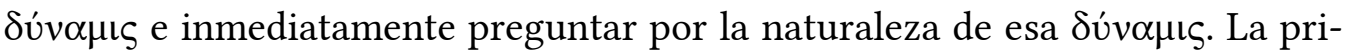
mera posibilidad que se plantea es identificarla con la materia, pero entonces parecen poder aplicarse los mismos argumentos esgrimidos antes respecto de los cuerpos. La segunda posibilidad es precisamente que esa $\delta \dot{v} \alpha \mu \iota \varsigma$ sea material ( $(" v v \lambda o v)$. La diferencia entre la ű $\lambda \eta$ y lo $\varepsilon ้ v v \lambda o v$ es explicitada en términos de participación: lo material es aquello que participa de la materia. A continuación, entonces, se interroga por esto que participa de la materia ( $\tau$ ò $\mu \varepsilon \tau \varepsilon \dot{\varepsilon} \chi o v \tau \tilde{\eta} \varsigma$ ü $\lambda \eta \varsigma)$ y se dan dos posibilidades: que sea ü $\lambda \eta$ o $\alpha \ddot{u} \lambda$ ov. Las dos posibilidades se rechazan puesto que la participación implica justamente que no se es aquello de lo que se participa pero que, a la vez, se tiene una relación con ello: por un lado, lo que participa de la materia no puede ser materia ya que justamente se dijo

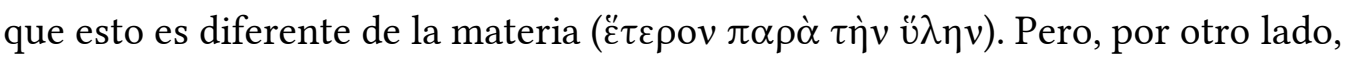
si esto que participa de la materia es inmaterial, entonces, hay que descartar que sea cuerpo. La última afirmación ("pues todo cuerpo $(\sigma \tilde{\omega} \mu \alpha)$ es material

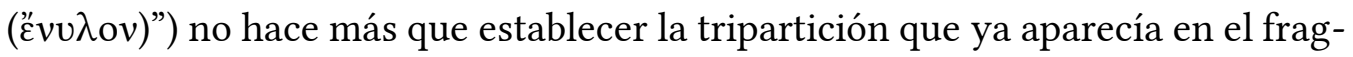

${ }^{26}$ E⿺̉ $\delta \dot{\varepsilon} \lambda \varepsilon \dot{\gamma} \gamma$

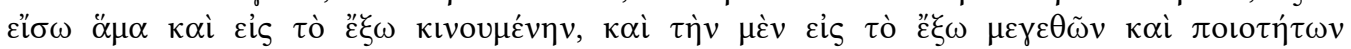

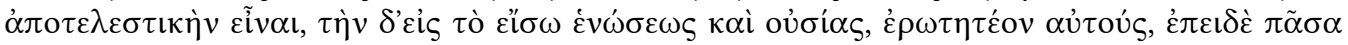

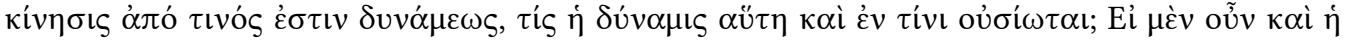

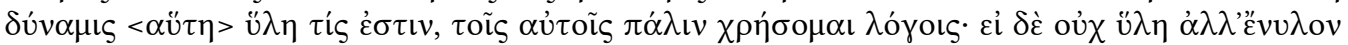

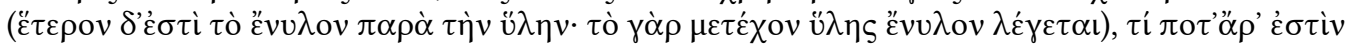

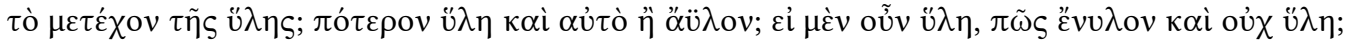

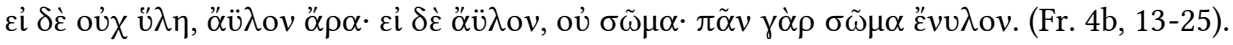

${ }^{27}$ La doctrina del $\tau$ óvo atribuida a Cleantes aparece en SVF II, 451. Una referencia a los movimientos de expansión ("hacia a fuera") y de contracción ("hacia adentro") se encuentra en SVF II, 452. 
mento 4a entre la materia, el cuerpo (= lo material) y lo incorpóreo (= lo inmaterial), ahora identificado con el alma. Por otra parte, si en el fragmento 4a se hacía hincapié en la relación de "sostenimiento" ( $\alpha \tau \varepsilon \dot{\chi} \chi \omega)$ de lo incorpóreo respecto de los cuerpos, en este fragmento $4 \mathrm{~b}$ a esta relación de "cohesión" ( $\sigma v v \varepsilon ́ \chi \omega)$ que establece el vínculo del cuerpo material con lo inmaterial/incorpóreo, se suma la relación de participación que establece el vínculo del cuerpo material con la materia. De este modo, quedan más completas las relaciones mutuas entre el alma incorpórea, el cuerpo y la materia.

A partir de aquí el texto de Nemesio incorpora doctrinas y argumentos que no estaban para nada mencionados en el fragmento citado por Eusebio. En primer lugar, se expone un argumento tendiente a negar que el alma sea cuerpo puesto que, si lo fuera, sería tridimensional. Esta corporeidad del alma basada en su tridimensionalidad se rechaza alegando que ella por sí misma ( $\left.\kappa \alpha \theta^{\prime} \dot{\varepsilon} \alpha v \tau \dot{\alpha}\right)$ es incorpórea y carece de dimensiones ( $\alpha \delta$ เ $\alpha \sigma \tau \alpha \tau$ os), pero se reconoce que por accidente ( $\alpha \tau \dot{\alpha} \sigma v \mu \beta \varepsilon \beta \eta \kappa o ́ \varsigma)$ sí es de tres dimensiones, aunque de un modo indirecto: por ser considerada en lo cuerpos en los que se da la tridimensionalidad. ${ }^{28}$

Luego, el testimonio de Nemesio alude a la doctrina platónica que considera al alma como principio del movimiento ${ }^{29}$ y distingue entre lo animado ( $\varepsilon \mu \psi v \chi \circ v)$, es decir, aquello que es movido por el alma "desde adentro"

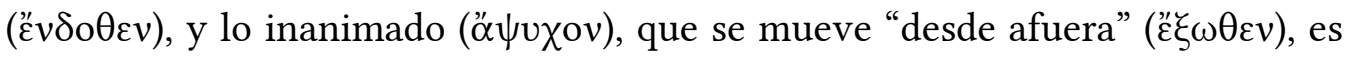
decir, a causa de otro cuerpo. En el primer caso, se trata de un alma que mueve a un cuerpo; en el segundo, se trata de un cuerpo que mueve a otro cuerpo desde el exterior. Si el alma fuera cuerpo, debería pertenecer a alguna de estas dos

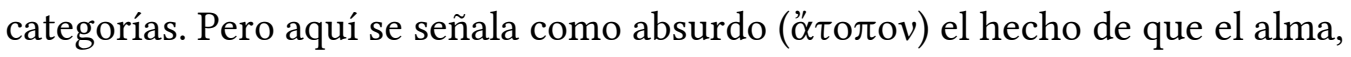
que es aquello por cuya presencia algo es $\varepsilon_{\mu} \mu \nu \chi 0 v$ y por cuya ausencia es ״ $\psi v \chi \chi v$, tenga alguna de estas características. Por lo tanto, se concluye que el alma no es cuerpo.

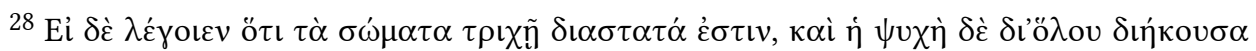

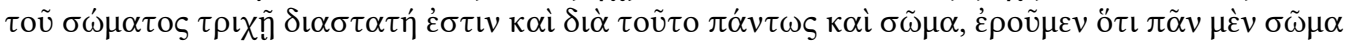

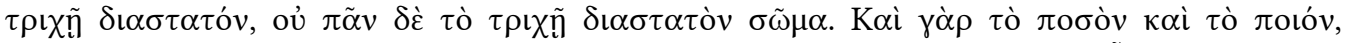

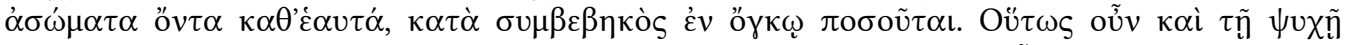

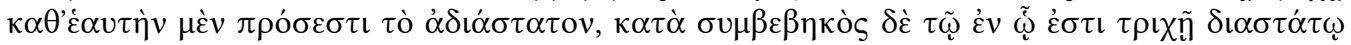

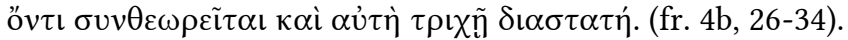

29 En Fedro 245c-e se dice que el alma, además de principio del movimiento, es semoviente. Sin embargo, en el fragmento 4a, Numenio decía que lo incorpóreo era inmóvil

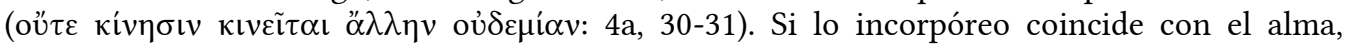
entonces parece que ella mueve sin moverse (al igual que el primer motor de Aristóteles, que

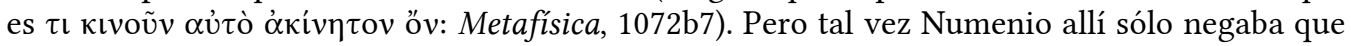
lo incorpóreo tuviera un movimiento análogo al de los cuerpos (generación, crecimiento y el resto de los cambios listados en los fragmentos 5 y 6). Volveré sobre esta caracterización del alma como principio del movimiento en la sección siguiente. 
Finalmente, el último argumento apela a la idea de los saberes $(\mu \alpha \theta \dot{\eta} \mu \alpha \tau \alpha)$ como alimentos del alma, presente en varios pasajes platónicos. ${ }^{30}$ Así, se plantean dos posibilidades. La primera es que el alma sí se alimente: si el alma se alimenta, lo hace con los saberes, que son incorpóreos. Pero, como ningún cuerpo puede alimentarse de algo incorpóreo, se concluye que el alma no es cuerpo. ${ }^{31}$ La otra posibilidad es que el alma no se alimente: si el alma no se alimenta, pero, en el caso de todos los seres vivos, ${ }^{32}$ su cuerpo sí se alimenta, de este modo también se concluye que el alma no es cuerpo. Como partiendo de cualquiera de los dos enunciados que conforman la contradicción (que el alma se alimente o que el alma no se alimente) se arriba a la misma conclusión, de este modo, ella queda demostrada. ${ }^{33}$

Si tomamos los elementos concordantes de los fragmentos 4a y $4 \mathrm{~b}$ llegamos a la conclusión de que es el alma aquel principio incorpóreo e inmaterial que da estabilidad a los cuerpos, que los "sostiene" o "contiene", los hace ser eso que son. Así, por una parte, los cuerpos participan de la materia y, en ese sentido, son "materiales", pero también están configurados por un alma que los hace ser "animados", es decir, los dota de un cierto movimiento interior. Vemos, de este modo, que el alma parece ser a la vez principio de estabilidad pero también de un cierto movimiento. Por otra parte, si bien las categorías de "material" y "animado" parecen coincidir en la caracterización de los cuerpos, veremos la dificultad de encontrar un correlato para lo inanimado ( $\propto$ « $\psi \chi \chi 0 v)$ que, en principio, parecería estar más bien asociado a la materia.

El siguiente cuadro pretende dar cuenta de los elementos presentes en los fragmentos analizados:

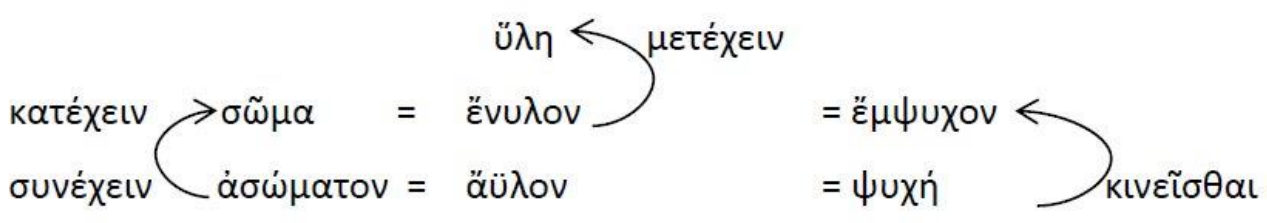
(Heinze).

30 Fedón 84a7-8 y Fedro 247d y 248b-c.

${ }^{31}$ Este argumento es atribuido explícitamente a Jenócrates y constituye el fragmento 66

${ }^{32}$ Creo que es importante que se haga esta aclaración, porque hay muchas cosas que son corpóreas, pero que no necesitan alimentarse.

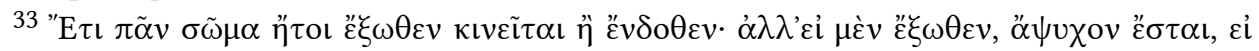

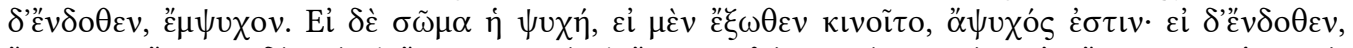

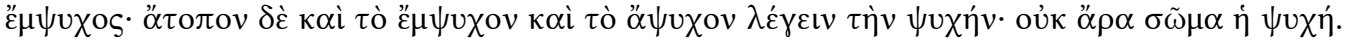
"Е

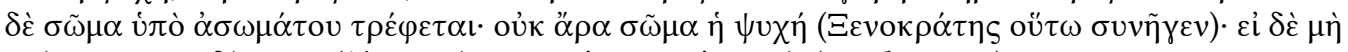

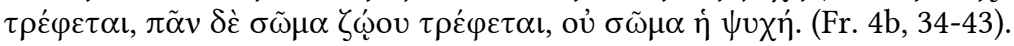




\title{
3. EL ALMA COMO PRINCIPIO DE MOVIMIENTO
}

\section{a) Materia, movimiento desordenado y alma mala: el fragmento 52}

Sobre el final del fragmento $4 \mathrm{~b}$, como vimos, aparecía la concepción del alma como principio del movimiento de los cuerpos animados. En un pasaje del comentario In Timaeum de Calcidio, Numenio apelará nuevamente a esta doctrina platónica pero ahora para explicar la vinculación entre el alma y el mal, nuevamente en contraposición a la explicación estoica del origen de los males en el mundo.

El testimonio sobre Numenio aparece en los parágrafos 295 a 299 del comentario de Calcidio (fragmento 52) y se refiere principalmente a su doctrina sobre la materia (silva), identificada allí con la duitas indeterminata, la neccesitas y también con la maligna anima de la que habló Platón en el libro X de Leyes. ${ }^{34}$ Esta última identificación, que es la que nos interesa en este trabajo, aparece en los parágrafos 296 y 297 de la mano de la crítica a los estoicos. Dice Calcidio:

\begin{abstract}
Así pues, Pitágoras -dice Numenio- también piensa que la materia es fluida y sin cualidad pero no, sin embargo, como los estoicos, de naturaleza intermedia entre lo próximo a los bienes y males, a lo que estos [los estoicos] llaman 'género indiferente', sino completamente perjudicial (plane noxia). Puesto que dios, como también le parece a Platón, es principio y causa de los bienes, la materia, de los males y, en cambio, lo que es a partir de la forma y la materia, [es] indiferente: por lo tanto, no [es] la materia sino el mundo, que es moderado a partir de la bondad de la forma y la maldad de la materia; en fin, ha sido producido a partir de la providencia y la necesidad según las máximas de los antiguos teólogos. ${ }^{35}$
\end{abstract}

La materia, como vimos en el fragmento 3, es fluida y, al igual que para los estoicos, ella carece de cualidad (sine qualitate). Sin embargo, que carezca de cualidad no quiere decir que sea un género indiferente (indifferens), es decir, una naturaleza intermedia entre el bien y el mal, como lo es para los estoicos. Para Numenio la materia es completamente perjudicial (plane noxia). El principal punto de la crítica de Numenio a los estoicos, que se repite a continuación en el texto (líneas 44 a 75), parece ser el derivar del carácter carente de cualidad

\footnotetext{
34 Jourdan 2014 y Dörrie-Baltes 1996, 162-173 analizan exhaustivamente este texto.

35 Igitur Pythagoras quoque, inquit Numenius, fluidam et sine qualitate silvam esse censet nec tamen, ut Stoici, naturae mediae interque bonorum malorumque viciniam, quod genus illi appellant indifferens, sed plane noxiam. Deum quippe esse -ut etiam Platoni videtur- initium et causam bonorum, silvam malorum, at vero quod ex specie silvaque sit, indifferens, non ergo silvam, sed mundum ex speciei bonitate silvaeque malitia temperatum; denique ex providentia et necessitate progenitum veterum theologorum scitis haberi. (fr. 52, 33-43).
} 
de la materia el hecho de que no sea ni buena ni mala, es decir, indiferente. Para Numenio, en cambio, una cosa no se sigue de la otra: la materia es sin cualidad y a la vez mala: la maldad de la materia no es un atributo que la cualifica sino una característica esencial (así como la bondad respecto de dios, el otro principio contrapuesto a la silva).

Este punto de discrepancia con el estoicismo es a su vez un punto de coincidencia con Platón, quien al igual que Pitágoras y Numenio, considera que dios es causa y principio de los bienes y la materia de los males (deus ... initium et causa bonorum, silva malorum).${ }^{36}$ Por último, y nuevamente en contraposición a la doctrina estoica, Numenio afirma que el mundo (mundus) es justamente lo indiferente (indifferens), pero no, como para los estoicos, porque no es ni bueno ni malo, sino porque es bueno y malo, dado que está compuesto, "temperado" o "moderado" (temperatus), a partir de la bondad de la forma (species) impuesta por dios y de la maldad de la materia o, para decirlo en los términos de los antiguos teólogos, es producido a partir de la providencia (providentia) y la necesidad (necessitas). ${ }^{37}$ Así, el mundo en lugar de ser una unidad, tal como lo era para los estoicos, es una suerte de reunión de dos órdenes: uno derivado del principio material y otro del principio divino. Aquello derivado de dios es identificado con la bondad y con la forma, aquello derivado de la materia es identificado con el mal y, en principio, con lo corpóreo.

El siguiente parágrafo se ocupa del punto central de la crítica a la posición estoica que, según Numenio, no puede precisamente dar cuenta de la existencia del mal. Dice:

Así pues, que la materia es informe y carente de cualidad tanto los estoicos cuanto Pitágoras lo consienten; pero Pitágoras [dice] también [que es] mala; los estoicos, [que es] ni buena ni mala. Por eso cuando algunos, en el curso del camino, se encuentran con males, al preguntarse ‘¿de dónde, pues, [vienen] los males?', [dicen] que son causados por la perversidad que ha de ser la simiente de los males. Pero no explican todavía de dónde [viene] la perversidad misma, puesto que entre ellos los principios de las cosas son dos: dios y materia: dios, bien sumo y sobresaliente, la materia -como piensan- ni bien ni mal. En cambio Pitágoras no teme asistir a la verdad aunque con aseveraciones asombrosas y que van contra la

36 Esta correspondencia axiológica de ambos principios está muy marcada en los

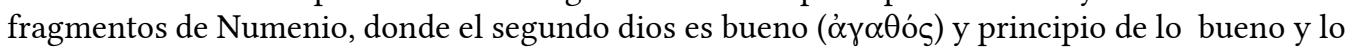
bello en el mundo y, a su vez, deriva su bondad del primer dios que, por eso, es $\tau$ ò $\alpha \gamma \alpha \theta$ óv y $\alpha \dot{\tau} \tau$ ó́ $\gamma \alpha \theta$ ov (frags. 16, 19 y 20).

37 "Theologos vocat Moysen, Orpheum, Pythagoram, Pherecydem, Homerum": Leemans 1937, 93, n. 6. Van Winden 1965, 105 y 111 añade a Hesíodo. Petty 2012, 214, por su parte, los

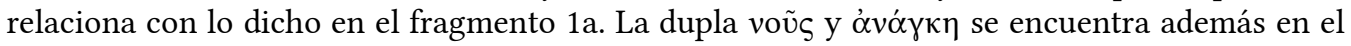
Timeo de Platón (47e4-5 y 48a1) y reaparecerá sobre el final de este testimonio de Calcidio. 
opinión de los hombres. Él dice que, al existir la providencia, también necesariamente subsisten los males, a causa de que existe la materia, y al mismo tiempo está dotada de maldad. Porque, si el mundo [es] a partir de la materia, sin duda está hecho de una naturaleza maligna que existe antes. ${ }^{38}$

Nuevamente, la base del acuerdo entre Pitágoras y los estoicos (que la materia es sin forma y sin cualidad) se rompe cuando estos últimos deducen, de esta carencia de cualidad, que la materia no debe ser identificada ni con el bien ni con el mal. Así, son incapaces de responder a la pregunta “unde mala?", ${ }^{39}$ porque no admiten que la materia sea el origen del mal y, si lo adjudican a una perversitas, aún tienen que responder de dónde proviene esa perversidad. Si ninguno de los dos principios a partir de los cuales se conforma el mundo es causa del mal, entonces no tienen modo de explicar su existencia, que, para Numenio, parece ser un hecho. La doctrina de Pitágoras, en cambio, aun cuando va en contra de la opinión de la mayoría, atribuye el mal a la materia, puesto que su naturaleza maligna (natura maligna) es principio del mal que hay en el mundo y, por eso, se dice que "existe anteriormente" (existens olim). ${ }^{40}$

Calcidio, a continuación, menciona quiénes coinciden con este punto de la doctrina pitagórica. Dice:

Y por esto Numenio alaba a Heráclito cuando censura a Homero, quien deseaba la destrucción y devastación de los males de la vida, porque no comprendía que le pareciera bien que el mundo fuera destruido, dado que la materia, que es fuente de los males, sería exterminada. También Numenio alaba a Platón, porque postula dos almas del mundo, una muy beneficiosa y la otra maligna, es decir, la materia, que, aunque fluya sin orden, sin embargo, puesto que se mueve con un movimiento interior y propio, es necesario que viva y que sea vivificada por un alma, según la ley de todas las cosas que

38 Silvam igitur informem et carentem qualitate tam Stoici quam Pythagoras consentiunt, sed Pythagoras malignam quoque, Stoici nec bonam nec malam. Dehinc, tamquam in progressu viae malis aliquot obuiis, perrogati: «unde igitur mala?», perversitatem seminarium malorum [fore] causati sunt. Nec expediunt adhuc unde ipsa perversitas, cum iuxta illos duo sint initia rerum, deus et silva, deus summum et praecellens bonum, silva, ut censent, nec bonum nec malum. Sed Pythagoras assistere veritati miris licet et contra opinionem hominum operantibus asseverationibus non veretur; qui ait existente providentia mala quoque necessario substitisse, propterea quod silva sit et eadem sit malitia praedita. Quod si mundus ex silva, certe factus est de existente olim natura maligna (fr. 52, 44-59).

${ }^{39} \mathrm{La}$ Enéada I 8 de Plotino trata sobre este mismo problema y titula precisamente Пó $\theta \varepsilon v$ $\tau \grave{\alpha} \kappa \alpha \kappa \alpha ́$.

40 No creo que aquí se esté aludiendo a una maldad anterior a la materia (que será identificada más adelante con el alma mala), como proponen Bakhouche 2011, 853-854, n. 1054 y Vimercati 2012, 90. 
se mueven con un movimiento originario (genuinus). Además, también ella es creadora y protectora de la parte pasible del alma, en la que hay algo corpóreo y mortal y semejante al cuerpo, así como la parte racional del alma tiene por responsable a la razón y a dios. Por consiguiente, este mundo está hecho a partir de dios y la materia. $^{41}$

Numenio está de acuerdo con la crítica que Heráclito le hace a Homero: ${ }^{42}$ desear la desaparición de los males del mundo es desear su destrucción, puesto que implicaría eliminar a la materia, que es uno de los principios que lo conforma. Pero más interesante aún es el acuerdo que se plantea con la doctrina platónica que postula dos almas: una muy buena (una beneficentissima) y la otra mala (maligna altera). La referencia aquí, como ya he adelantado, es al Libro X de Leyes donde Platón distingue un alma benéfica, que conduce al mundo del mejor modo y un alma mala que, de guiar el movimiento del cosmos, lo haría de manera alocada $(\mu \alpha \nu \iota \kappa \tilde{\omega} \varsigma)$ y sin orden $(\dot{\alpha} \tau \dot{\alpha} \kappa \tau \omega \varsigma) .{ }^{43} \mathrm{Y}$ Numenio asocia esta alma mala con la materia (scilicet silva). ${ }^{44}$

La materia, como ya se dijo en el parágrafo anterior y aquí se repite, es fluida y este "flujo" es caracterizado como un motus intimo porprioque que la mueve. Como todo lo que se mueve a sí mismo, con un motus genuinus, debe tener un alma que sea el principio de ese movimiento interno, entonces de este modo se explica que Platón, en la interpretación de Numenio, haya postulado un alma mala, en virtud de su identificación previa de la materia con el movimiento desordenado (incondite).$^{45} \mathrm{El}$ pasaje finalmente señala otro punto en el que Numenio acuerda con Platón: la materia es autrix et patrona de la parte

41 proptereaque Numenius laudat Heraclitum reprehendentem Homerum, qui optaverit interitum ac uastitatem malis vitae, quod non intellegeret mundum sibi deleri placere, siquidem silva, quae malorum fons est, exterminaretur. Platonemque idem Numenius laudat quod duas mundi animas autumet, unam beneficentissimam, malignam alteram, scilicet silvam, quae, licet incondite fluctuet, tamen, quia intimo proprioque motu movetur, vivat et anima convegetetur necesse est lege eorum omnium quae genuino motu movetur; quae quidem etiam patibilis animae partis, in qua est aliquid corpulentum mortaleque et corporis simile, auctrix est et patrona, sicut rationabilis animae pars auctore utitur ratione ac deo. Porro ex deo et silva factus est iste mundus. (fr. 52, 59-75).

42 Van Winden 1965, 113 analiza esta referencia de Heráclito a Homero.

43 Leyes, 896e4-6 y 897d1.

44 Sigo aquí la edición de Waszink 1962, 299, con la que acuerda Van Winden 1965, 113, pero de la que se aparta Bakhouche 2011, 854, n. 1057 siguiendo la conjetura de Theiler ya propuesta antes por Beutler, que lee aquí: scilicet silvae. Esta variación del texto de la tradición manuscrita se adopta porque se interpreta que aquí Numenio habla de un alma mala que es el alma "de la materia", tal como el segundo grupo de platónicos mencionados por Calcidio en el parágrafo 300, que afirma que la materia está dotada de un alma (silva anima praedita).

45 Timeo 30a es el pasaje que está en la base de esta caracterización numeniana de la materia como un movimiento desordenado. 
pasible del alma, que está relacionada con lo material, mortal y corpóreo. Por su parte, dios y su ratio (ratio ac deus) es el auctor de la parte racional del alma.

Si se identifica a la materia con el movimiento desordenado y se postula un alma mala que explique ese movimiento, entonces parece lógica la necesidad de postular dos almas, tanto en el cosmos cuanto en el individuo: si bien el texto de Calcidio habla de dos partes del alma humana, quizás Numenio fue más enfático en este sentido y se atrevió a postular directamente dos almas diferenciadas. Porfirio parece dar cuenta de esta doctrina.

\section{b) Las dos almas: el fragmento 44}

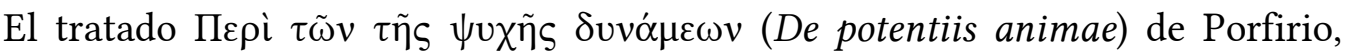
conservado gracias a las citas que el célebre compilador del siglo V Juan Estobeo realiza en la sección del Anthologium dedicada al alma, ${ }^{46}$ incluye dos pasajes sobre Numenio (los fragmentos 44 y 45) y uno de ellos (el fragmento 44) trata sobre las distintas posiciones respecto de la cantidad de partes ( $\mu \varepsilon ́ \rho \eta)$ del alma: los estoicos distinguieron ocho partes del alma; en cambio, Platón y Aristóteles postularon sólo tres. Y a continuación, Porfirio menciona la posición de Numenio, que parece ser la más radical al respecto. Dice:

Otros, entre los que [está] Numenio, creen que nosotros tenemos, no tres ni tampoco dos partes de un alma única: la [parte] racional

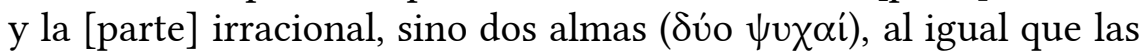

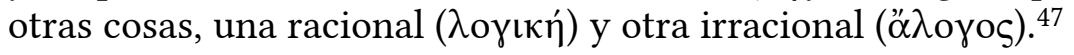

A diferencia del testimonio de Calcidio, según Porfirio Numenio se contaba entre quienes, en lugar de atribuir distintas partes a un alma unitaria, consideraba que tenemos directamente dos almas diferentes: una racional (

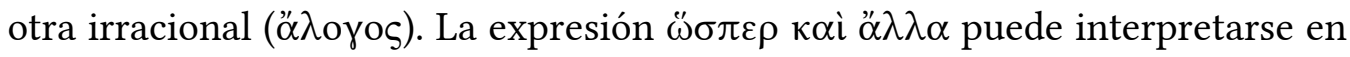
dos sentidos: las dos almas podrían corresponderse con el resto de nuestros miembros y órganos sensoriales, que también son pares, ${ }^{48} \mathrm{o}$ el resto de los seres

${ }^{46}$ Estobeo, Anthologium, I, 49.

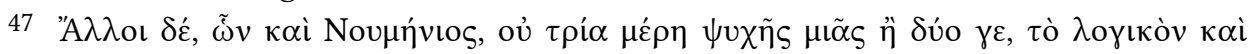

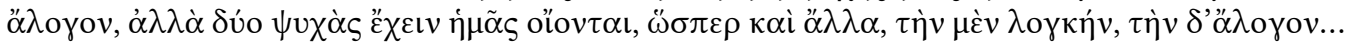

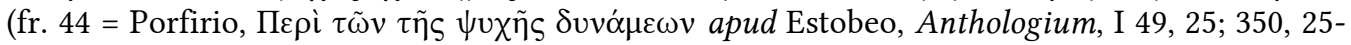
$351,1)$.

48 Esta es la interpretación que, en general, adoptan los traductores de este fragmento: Des Places 1973, 122, n. 1 al fr. 44 y García Bazán 1991, 289, n. 142. Petty 2012, 85 traduce de modo más indefinido "as in others respects". Pero, de hecho, ״ $\alpha \lambda \lambda \alpha$ es una conjetura de Wachsmuth, el editor de la obra de Estobeo, que corrige el $\alpha$ $\alpha \lambda$ or de los manuscritos, puesto que justamente interpreta que " $\alpha \lambda \lambda \alpha$ sunt aures, oculi, manus, etc." (ver el aparato crítico a este pasaje en la p. 350). Sin embargo, según la otra interpretación que aquí propongo, el $\alpha \lambda \lambda \lambda o r$ de los manuscritos tendría también sentido. 
del mundo podrían tener dos almas, al igual que nosotros. Esta segunda interpretación sería más amplia, ya que señalaría la duplicidad que gobierna en todo

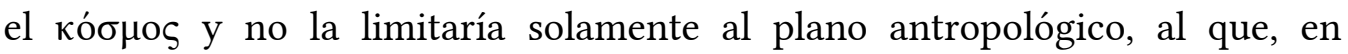
principio, parece estar restringido este testimonio. Sin embargo, esta interpretación no implicaría necesariamente adjudicar la racionalidad a todos los seres, sino simplemente una dualidad intrínseca que estaría representada por estas dos almas; sólo en el hombre el resultado de la acción del principio divino sería el alma racional (entendida en sentido estricto). No obstante, esta interpretación

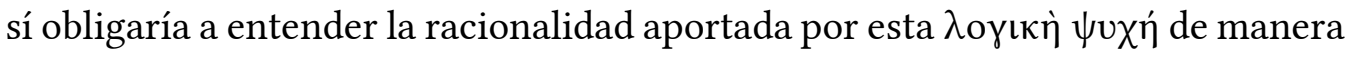
más amplia, ya que aludiría a la acción del principio divino que opera en toda la realidad, de manera similar al $\lambda$ ójos estoico; aunque, a diferencia de lo que

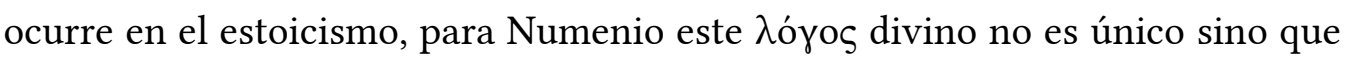
coexiste con el principio material, representado aquí por la $\alpha \lambda$ oyos $\psi v \chi \eta ́$.

\section{CONSIDERACIONES FINALES: ALMA, MOVIMIENTO Y PERMANENCIA}

La caracterización del alma como principio de estabilidad y permanencia, por un lado, y como principio de movimiento, por otro, parece apuntar a la diferenciación entre el principio divino y el principio material. Sin embargo, el fragmento 15, citado por Eusebio en el Libro XI de su PE, desdibuja esta distinción entre movimiento y permanencia. Como parte de una serie de pasajes en los que Numenio distingue entre un primer dios y un segundo dios, este fragmento afirma:

Y estas son las vidas, por un lado, la del dios primero, por otro, la del segundo. Evidentemente el dios primero será el que está firme, el segundo contrariamente es el que se mueve. Ciertamente el primero [está] en torno a los inteligibles y el segundo [está] en torno a los inteligibles y sensibles. ${ }^{49}$

Numenio distingue dos dioses de acuerdo a dos tipos de vida: el primero está

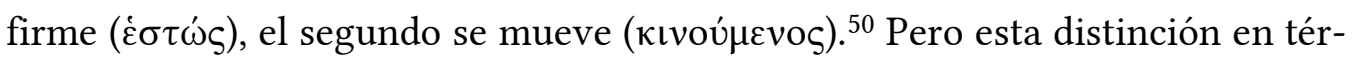
minos de movimiento y reposo se complementa con una diferenciación ulterior:

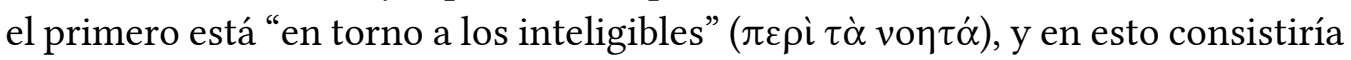
su estabilidad, y el segundo, "en torno a los inteligibles y sensibles" ( $\pi \varepsilon \rho \grave{i} \tau \grave{\alpha}$

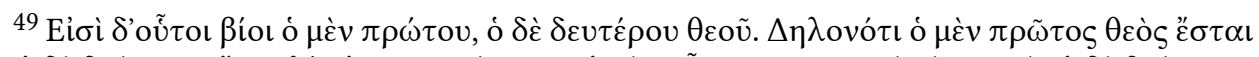

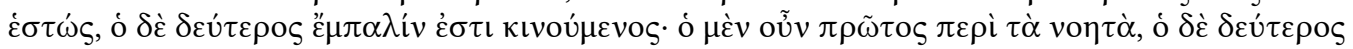
$\pi \varepsilon \rho \grave{~} \tau \grave{\alpha} v o \eta \tau \grave{\alpha} \kappa \alpha \grave{~} \alpha i \sigma \theta \eta \tau \dot{\alpha}$. (fr. 15, 2-5).

50 Permanencia y movimiento son un par de contrarios con una larga historia en la tradición filosófica (baste mencionar, al respecto, su presencia entre los cinco géneros mayores postulados por Platón en el Sofista 254d y ss.) y aquí Numenio los usa como una variable más para distinguir al primer dios del segundo.
} 


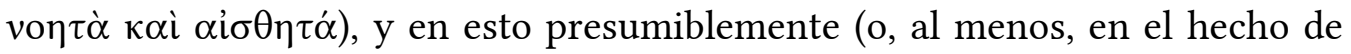
estar $\pi \varepsilon \rho \grave{i} \tau \dot{\alpha} \alpha i \sigma \theta \eta \tau \dot{\alpha})$ consistiría su movimiento. Sin embargo, la segunda parte del fragmento nos sorprende con una importante precisión:

Pero no te asombres si dije esto, pues escucharás algo aún más asombroso. Pues, frente al movimiento que pertenece al segundo, digo que el reposo que pertenece al primero es un movimiento connatural, a partir del cual el orden del cosmos, la permanencia, la eternidad y la salvación se derraman sobre la totalidad. ${ }^{51}$

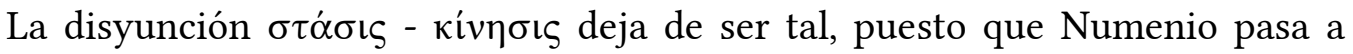
caracterizar la $\sigma \tau \alpha \dot{\alpha} \sigma \iota \varsigma$ del primer dios como un "movimiento connatural"

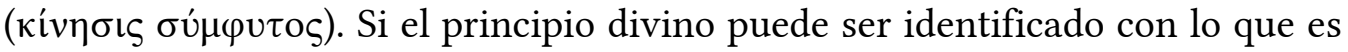



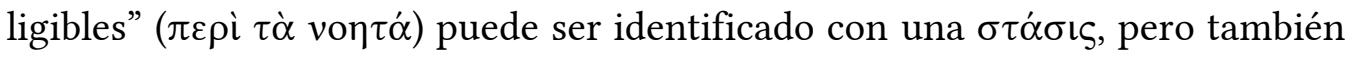

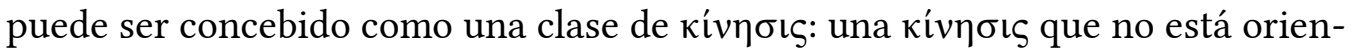
tada hacia otra cosa sino hacia la propia naturaleza y, por eso, es caracterizada como $\sigma u ́ \mu \varphi v \tau o \varsigma$. Y a partir de este movimiento connatural del primer dios, la totalidad de las cosas reciben las características que la emparentan con lo

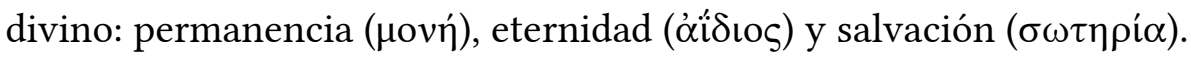

Por su parte, la duplicidad presente en el segundo dios, que está $\pi \varepsilon \rho \grave{~} \tau \grave{\alpha}$


señala en los fragmentos 11, 12 y 18 y que permitiría ver aquí una distinción entre el segundo y un tercer dios, presente explícitamente en dos testimonios del comentario In Timaeum de Proclo (fragmentos 21 y 22). Si bien el análisis de estos textos supera los límites del presente trabajo, la conclusión de que el plano divino articulado en tres instancias presenta un movimiento que es fuente de permanencia de lo sensible parece estar en consonancia con lo dicho en los fragmentos $4 \mathrm{a}$ y $4 \mathrm{~b}$ respecto de lo que es y del alma. ${ }^{53}$

La distinción fundamental, entonces, para Numenio no parece ser entre el movimiento y la permanencia, entre cambio y estabilidad, sino entre dos tipos de movimiento: uno que garantiza la estabilidad y la racionalidad de lo real y el otro que implica irracionalidad y desorden. Y esta diferenciación se corresponde

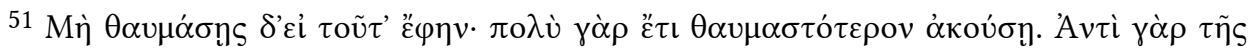

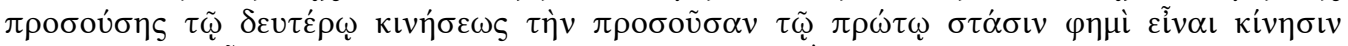

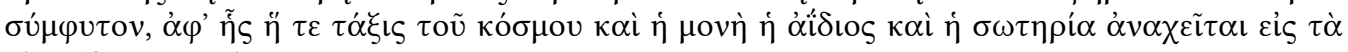
ö $\lambda \alpha$. (fr. 15, 5-10).

${ }^{52}$ Esta idea aparece claramente en los fragmentos 5 a 8 de Numenio.

${ }^{53}$ Para un análisis de la doctrina de los tres dioses, la relación del tercer dios con el alma y la noción de "vida" en Numenio, ver Müller 2015. 
con los dos principios afirmados por Numenio: dios y materia. Si en el estoicismo estos principios son caracterizados como cuerpo, en Numenio en cambio lo corpóreo, material y animado es más bien el resultado de la interacción entre estos principios que parecen ser anímicos, en tanto están caracterizados en términos de movimiento. Y precisamente en el encuentro e interacción entre ambos movimientos anímicos se ubican el mundo y también el hombre, en tanto ser corpóreo y animado. ${ }^{54}$

\section{BIBLIOGRAFIA}

Bakhouche, B. 2011. Calcidius. Commentaire au Timée de Platon. Paris : Vrin.

Barnard, L. W. 1972. "The Father of Christian Anthropology." Zeitschrift für die neotestamentliche Wissenschaft 63: 254-270.

Boeri, M. - Salles, R. 2014. Los filósofos estoicos. Ontología, lógica, física y ética. Sankt Augustin: Academia Verlag.

Des Places, É. 1973. Numénius. Fragments. Paris: Les Belles Lettres.

Des Places, É. 1987. Eusèbe de Césarée. La préparation évangélique. Livres XIV$X V$. Paris: Éditions du Cerf.

Dodds, E. 1960. "Numenius and Ammonius." En Les sources de Plotin [Entretiens sur l'antiquité classique, tome V], 1-61. Genève, Vandoeuvres: Fondation Hardt.

Dörrie, H. - Baltes, M. 1996. Der Platonismus in der Antike: Der philosophische Lehre des Platonismus [1]: Einige grunlegende Axiome / Platonische Physik (im antiken Verständnis) I, Band 4. Stuttgart-Bad Cannstatt: FrommannHolzboog.

Dörrie, H. - Baltes, M. 2002. Der Platonismus in der Antike: Der philosophische Lehre des Platonismus [3]: Von der »Seele « als der Ursache aller sinnvollen Abläufe, Band 6, 1-2. Stuttgart-Bad Cannstatt: Frommann-Holzboog.

García Bazán, F. 1991. Oráculos Caldeos; Numenio de Apamea: Fragmentos y Testimonios. Madrid: Gredos.

Jourdan, F. 2014. "La matière à l'origine du mal chez Numénius (fr. 43 et 52 Des Places)." Philosophie antique 14: 185-235.

Leemans, E.-A. 1937. Studie over den Wijsgeer Numenius van Apamea met Uitgave der Fragmenten. Bruxelles: Palais des Académies.

Mié, F. 2018. "Refining the Material Substance: Aristotle's Program in Metaphysics H1-5”. Síntesis. Revista de Filosofía I (2): 54-100.

54 Este artículo retoma una sección de mi tesis de doctorado dedicada al pensamiento metafísico de Numenio de Aparmea y financiada con una beca doctoral otorgada por CONICET. Agradezco las observaciones y correcciones de los evaluadores y muy especialmente a la editora de este número, Gabriela Rossi, por sus enriquecedores comentarios. Todos los errores son míos. 
Müller, G. 2015. "Qué es "lo que es viviente" (ö દ̇ $\sigma \tau \iota \zeta \tilde{\omega} o v)$ según Numenio de Apamea." Cuadernos de Filosofía 64: 9-22.

Petty, R.. 2012. Fragments of Numenius of Apamea. Westbury, Wiltshire: The Prometheus Trust.

Smith, A. 1993. Porphyrii Philosophi Fragmenta. Stuttgart-Leipzig: Teubner.

Van Winden, J. C. M. 1965. Calcidius on Matter. His Doctrine and sources. Leiden: Brill.

Vimercati, E. 2012. "La materia e il male in Numenio di Apamea." Filosofia e Teologia XXVI/1: 77-92.

Von Arnim, H. 1903-24. Stoicorum Veterum Fragmenta (= SVF). 4 vols. Leipzig: Teubner.

Wachsmuth, C. - Hense, O. 1884-1912. Ioannis Stobaei Anthologium. 5 vols. Berlin: Weidmann.

Waszink, J. H. 1962. Timaeus, a Calcidio translatus commentarioque instructus, Londres- Leiden: Brill. 